\title{
DESIGN FOR COMPOSITES: DERIVATION OF MANUFACTURABLE GEOMETRIES FOR UNIDIRECTIONAL TAPE LAYING
}

\author{
Voelkl, Harald; Kießkalt, Andreas; Wartzack, Sandro \\ Friedrich-Alexander-Universität Erlangen-Nürnberg
}

\begin{abstract}
Even though providing excellent specific stiffness and strength properties, high specific energy absorption and a great degree of design freedom, fibre-reinforced plastics still have to make their way into higher volume applications. Addressing the manufacturing challenges, particularly efficient production techniques are Automated Tape Laying (ATL) and Automated Fiber Placement (AFP), as pointed out by various studies and use cases. However, current Computer Aided Engineering approaches for optimised laminate design still lack the capability to produce results suitable for ATL/AFP. A new method for deriving tape courses from any finite element laminate optimisation result is presented and applied to a virtual demonstrator. An outlook is given on further necessities of extending current laminate optimisation approaches.
\end{abstract}

Keywords: Optimisation, Computational design methods, Design for X (DfX), Fibre Reinforced Plastics, Manufacturing

Contact:

Voelkl, Harald

Friedrich-Alexander-Universität Erlangen-Nürnberg

Engineering Design

Germany

voelk1@mfk.fau.de

Cite this article: Voelkl, H., Kießkalt, A., Wartzack, S. (2019) 'Design for Composites: Derivation of Manufacturable Geometries for Unidirectional Tape Laying', in Proceedings of the 22nd International Conference on Engineering Design (ICED19), Delft, The Netherlands, 5-8 August 2019. DOI:10.1017/dsi.2019.275 


\section{LAMINATE OPTIMISATION AND PRODUCTION TECHNIQUES: STATUS QUO AND CHALLENGES}

Even though providing excellent specific stiffness and strength properties, high specific energy absorption and a great degree of design freedom (Schürmann, 2007), fibre-reinforced plastics (FRP) still have to make their way into higher volume applications. According to a current study (Reden $e t$ al., 2018), production costs would be largely reduced by increasing the production rate. A significant part of cost reduction can be achieved through efficient production technologies - even more cost reduction than by cutting material costs in half. Already more than 25 years ago, aerospace companies claimed man-hours decrease of up to 70-85\% (Grimshaw M.N. et al., 2001) by using a particularly efficient production technique: Automated Tape Laying (ATL). It reduces cut-off largely, which is one of the main drivers of production costs for FRP parts; Figure 1 illustrates its working principle.

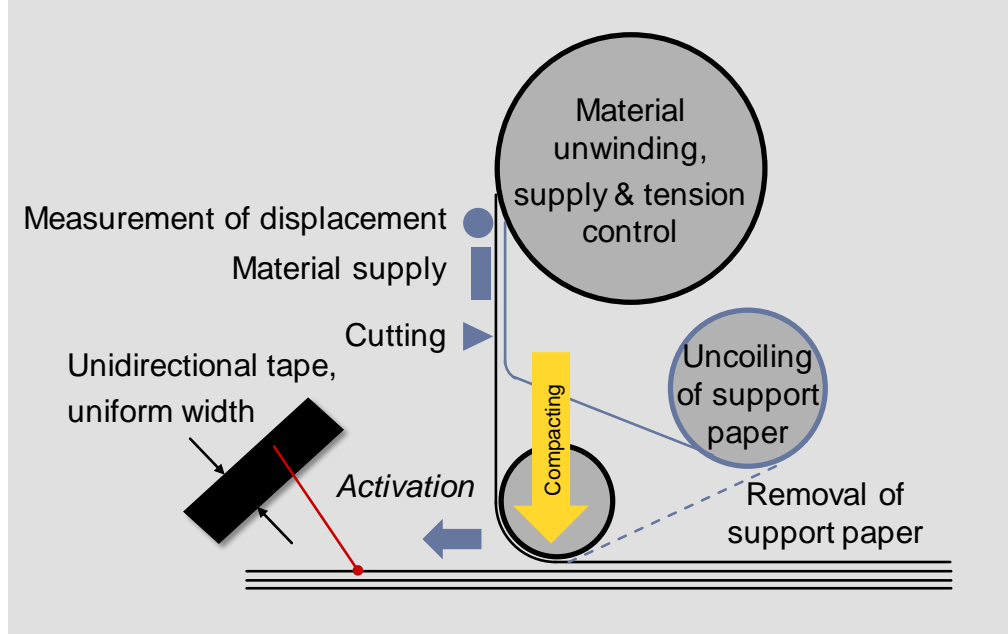

Figure 1. Automated tape laying (ATL) principle as in (Lengsfeld et al., 2015)

The ATL manufacturing process is based on sequential, contour-based, additive laying of fixed-width unidirectional tapes consisting of preimpregnated fibers (prepregs) (Lengsfeld et al., 2015). A separating support paper detaches the prepreg tapes from each other on the coil, which is removed before the actual draping takes place. Typical prepreg roll widths are 75, 150 and $300 \mathrm{~mm}$. Various machines exist with multiple axes of movement, which allow flat and contour (3D) tape laying, with contour angles depending on the machine used. While ATL is suitable for relatively flat surfaces, Automated Fiber Placement (AFP) offers more flexibility for curved surfaces laying narrow tows (up from $3.2 \mathrm{~mm}$ ) and, like ATL, requires similar information on tape courses. (Lukaszewicz et al., 2012) Considering such production possibilites, it is obviously desirable to count these in already during engineering design processes. Existing tools are able to obtain laminate designs fulfilling certain objectives. However, their layer geometries possess more or less arbitrary shapes (usually only manufacturable by hand lay-up), Figure 2 .
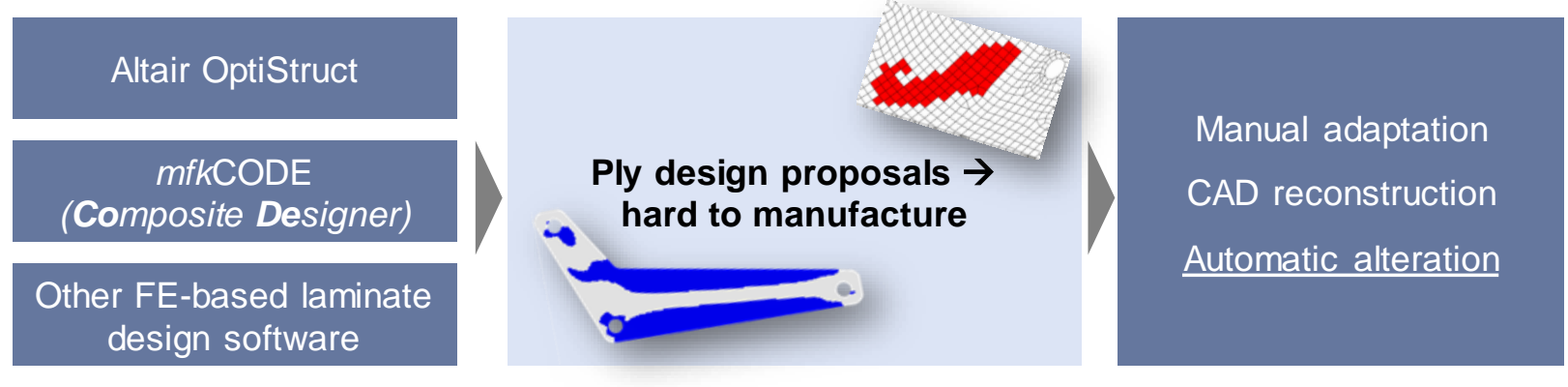

Figure 2. Challenge of finding proper layer geometries 
To overcome that limitation, this contribution exemplarily extends an existing approach for optimised laminate design with endless-fibre reinforced plastics, mfkCODE (Klein et al., 2015), to obtain layer geometries more suitable for the ATL/AFP processes.

\section{DERIVATION OF UNIDIRECTIONAL (UD) TAPE LAYING GEOMETRIES FROM OPTIMISED UD CLUSTERS}

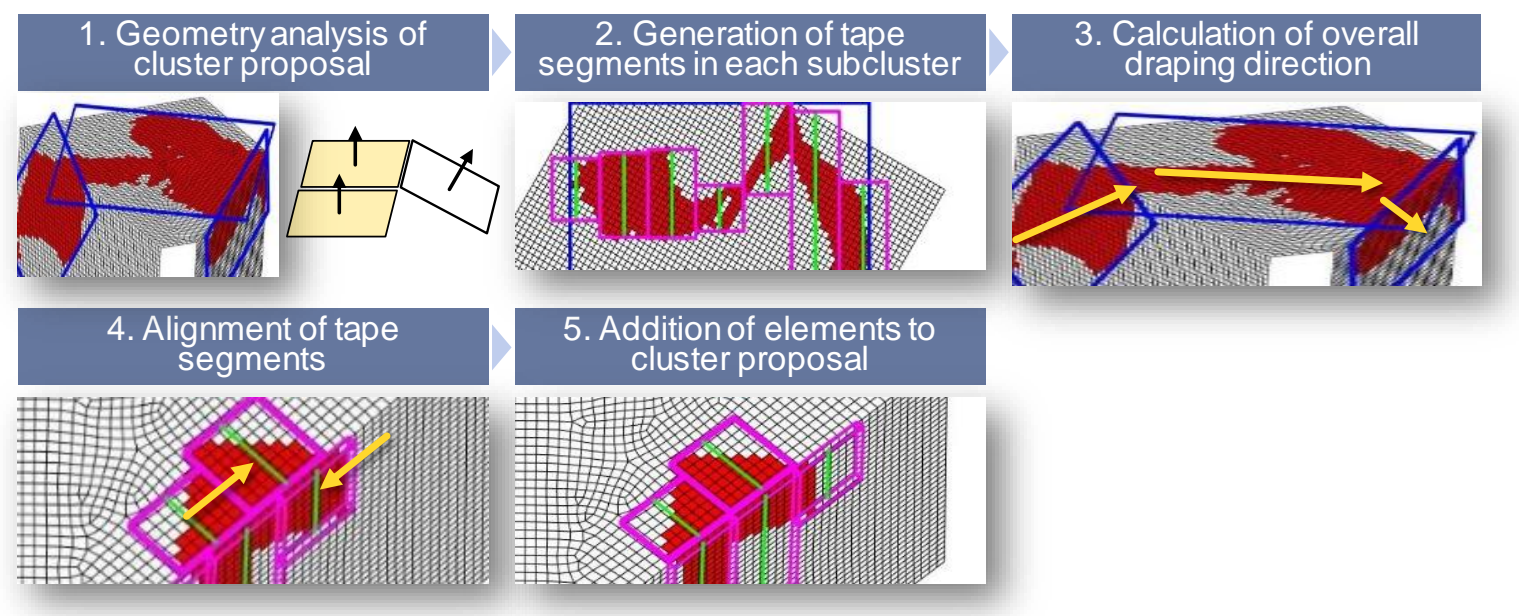

Figure 3. Steps for deriving tape laying geometries: 1. Geometry analysis (elements of cluster in red), 2. Tape segments, 3. Draping direction, 4. Segment alignment, 5. Result

Figure 3, Step 1 shows a typical result from a FE-based laminate optimisation tool for one ply: an optimised ply shape, defined by finite elements belonging to a certain region of similar fibre orientation ("cluster" in the following). Each element bears information about its fibre orientation, which obviously is (draping considered) the same as for all other elements in the ply. Threedimensional geometries are taken into account either by draping simulations or by "flipping" the orientation vectors according to element contours (curves or edges) into the same plane. The outer ply contours are largely irregular, depending on the ply clustering algorithm (or similar) used before.

In Step 2 of the new algorithm for UD tape laying (Figure 3), these plies are separated into nearly flat sub-plies according to geometry. The algorithm then separates these sub-plies into ATL tape segments of a pre-defined width, e.g. $75 \mathrm{~mm}$, considering the local fibre orientation. The overall tape-laying direction is further derived taking both geometry and fibre orientation into account (Step 3). The subplies' tape segments are aligned to each other to obtain a continuous laying path (Step 4). Finally, all newly found elements are added to the respective ply to allow for FE simulation of the adapted laminate afterwards (Step 5). In the following, the details of these steps are discussed in detail.

\subsection{Creation of sub-plies}

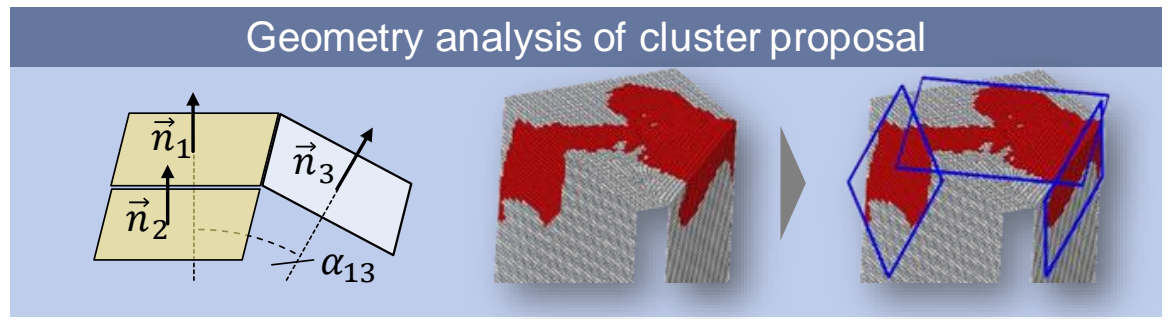

Figure 4. Geometry analysis - subclustering

Starting from an optimised ply shape, the algorithm clusters its elements by their normal vectors within a user-specified tolerance (Figure 4 on the left). Elements with normal vectors pointing in the same overall direction (i.e. $\alpha_{i j}<90^{\circ}$ ) and differing less than the tolerance threshold in angle are added to one local cluster, called sub-ply. Resulting sub-plies must also be coherent. That means that there must be a direct connection between elements, otherwise two sub-plies are generated, Figure 5 . 


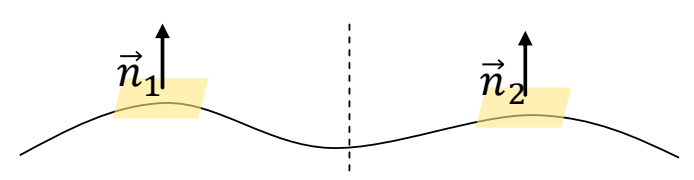

Figure 5. Two separate sub-plies despite normal vectors within similarity tolerance

\subsection{Generation of tape courses}

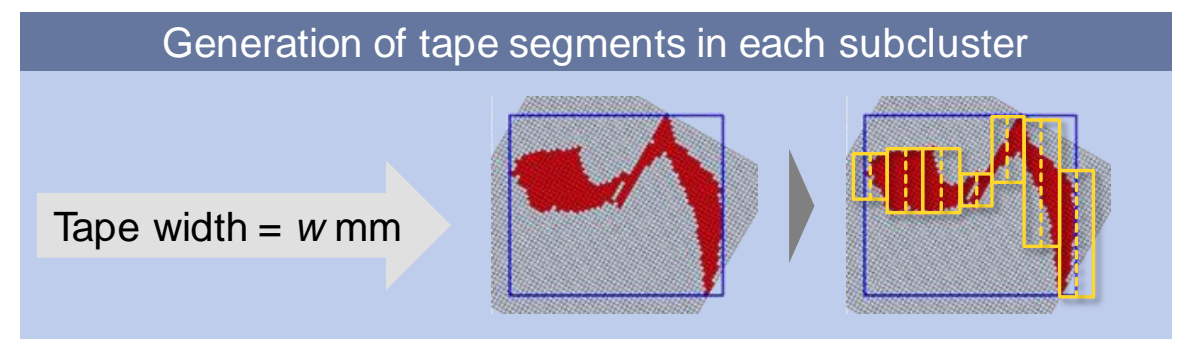

Figure 6. Generation of tape segments in each subcluster

After splitting up the original ply into the sub-plies, the algorithm divides these up into tape laying segments with a fixed width defined by manufacturing (Figure 6) using coordinate transformation of each (nearly-plane, depending on normal vector angle tolerance) sub-ply into the XY plane. In reality, there is a small gap between the individual tape courses to account for variations in placement due to tape tolerances and layup control (Lukaszewicz et al., 2012), which can be accounted for in the algorithm. Skipping this for simplicity, the tape courses are placed without gap with the optimised fibre orientation equidistantly. Obviously, there is no continuous tape course between individual subplies at this stage "over-edge".

\subsection{Overall laying direction}

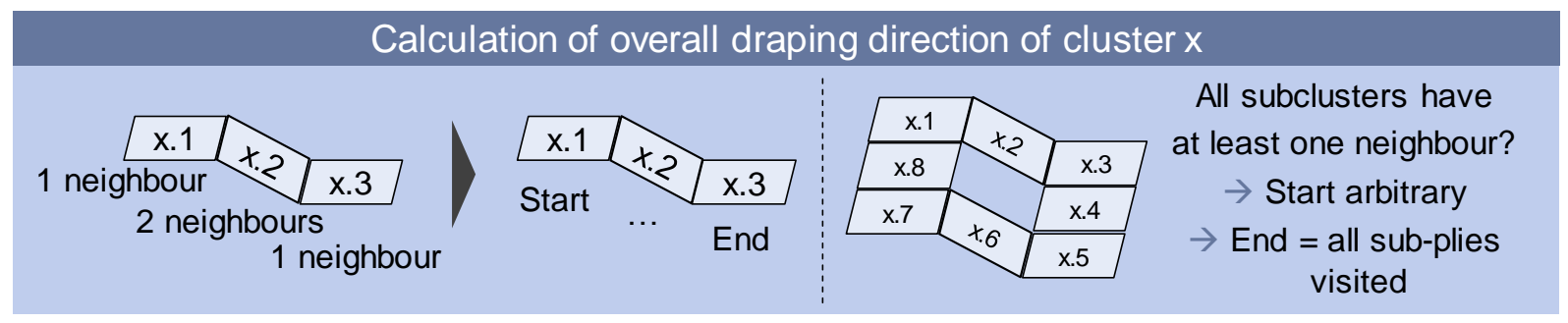

Figure 7. Calculation of overall laying direction

To connect the plane, taped sub-plies, the overall laying direction is calculated. First, the algorithm detects a starting sub-ply (where an ATL machine initiates the tape course). To achieve this, for each sub-ply all neighbour sub-plies are found by a separate algorithm "observing" the sub-ply outer contours' neighbouring elements. If a single sub-ply only possesses one neighbour, it qualifies as a starting or ending sub-ply (i.e., no sub-ply either "before" or "after", Figure 7 on the left). Running through consecutive neighbour sub-plies, starting from the first sub-ply's single neighbour onwards, an overall laying direction is defined until another single-neighbour sub-ply is found which marks the end of a tape course (Figure 7, left). In case there's no sub-ply with only one consecutive sub-ply ("neighbour"), e.g. the cluster being shaped as a ring (Figure 7, right), then an arbitrary sub-ply is used as starting sub-ply and this part of the algorithm stops as soon as all sub-plies are covered. 


\subsection{Alignment of tape courses}

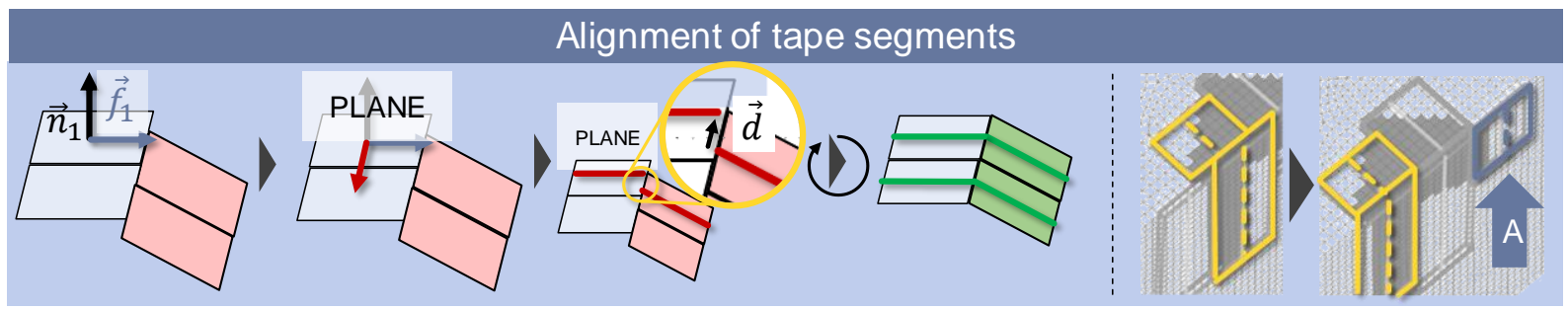

Figure 8. Alignment of tape segments

Following this overall laying direction, the individual tape courses of the sub-plies are aligned "overedge" to multiple, continuous, parallel tape courses (Figure 8). The algorithm stores the tape course information. The alignment is done by calculation of minimum distance between the lines by:

- (1) creating a plane perpendicular to the surface with its normal vector being the cross product between the direction vector of one of the courses $\overrightarrow{f_{1}}$ and its surface's normal vector $\overrightarrow{n_{1}}$ and

- (2) calculating the distances between the plane and all starting points of the lines of the next sub-ply (Hesse normal form). The beginning of the tape course belonging to the closest distance will be shifted towards the original tape course along vector $\vec{d}$ if its length is smaller than half tape-width.

- The "half tape-width" condition is necessary as, first, if shifting is farer than that, too much overlapping (even plies on top of each other) is possible; or, second, there's no "continuous" line at this crossing because the next sub-ply widens and needs more tape lines than the subply before (detail A in Figure 8 on the right).

- The whole process is repeated for all lines of the "current" sub-ply, then for all consecutive sub-plies.

Finally, the new elements within the tape courses are added to the initial ply element list including fibre direction information. Thus, the ply geometries generated here can be analysed in a FE simulation. After a short academic example in subchapter 2.5, the following use case in chapter 3 illustrates the whole process and focusses on mechanical performance of optimised, adapted results.

\subsection{Exemplary result of the algorithm}

Figure 9, finally, shows the algorithm applied to an academic example. Three sides of a cube with rounded transitions are subjected to various loads and boundary conditions, resulting in a chaotic optimised laminate with lots of different directions. The algorithm is capable of creating sub-plies and generation of tape courses within each; it then aligns the courses "over-edge" as far as possible. Overall results are shown in Figure 9 a) and these of an example ply in Figure 9 c). Figure 9 b) presents all modified plies; the exemplary modified single ply is shown in Figure 9 d).
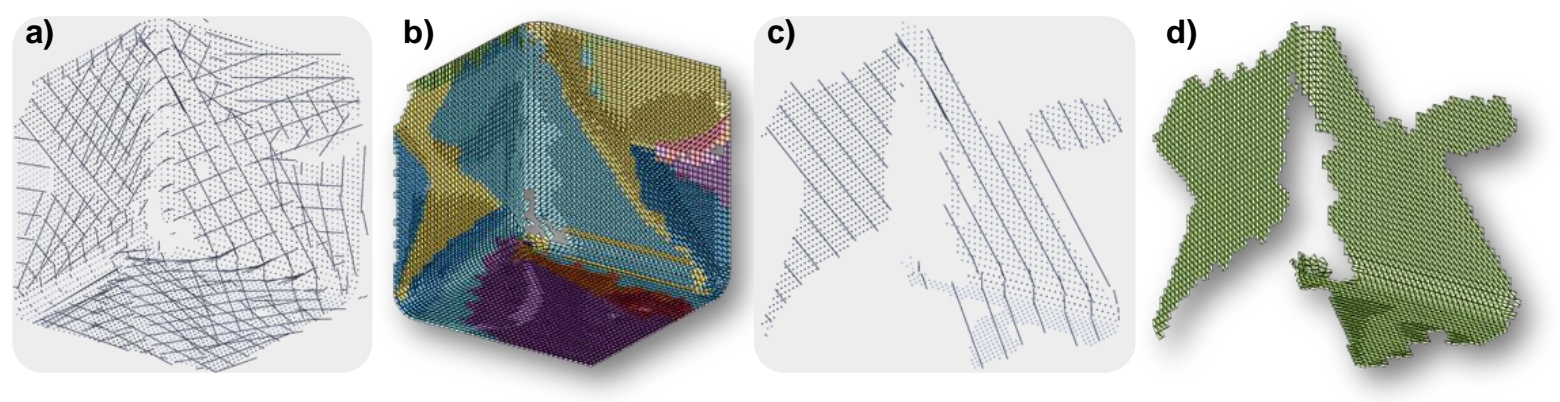

Figure 9. "Cube" example 


\section{USE CASE}

The demonstrator part for the following use case is presented in Figure 10. The seat of a glider is subjected to the load of a sitting pilot (approximately). The main purpose of the complicated load case is to provoke multiaxial stress states on a free-form geometry, leading to a complicated, optimised laminate with many layers and directions. This poses an interesting challenge for the ATL algorithm with tape placement along bent surfaces.

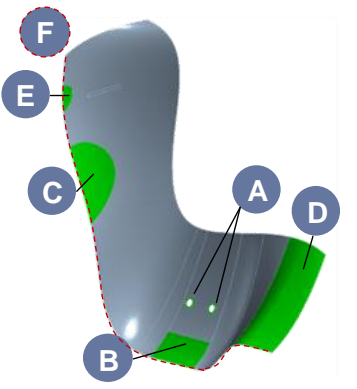
A: Fixed support
B: Force normal to area, $500 \mathrm{~N}$
C: Like B, $500 \mathrm{~N}$
D: Like B, $150 \mathrm{~N}$
E: Like B, $50 \mathrm{~N}$
$F$ : Symmetry constraint

Figure 10. Demonstrator part for use case

The optimisation problem in this chapter is specified as follows: minimize weight while keeping Puck failure criteria (both matrix and fibre) below 0.5 (strength) and maximum displacement below $2 \mathrm{~mm}$ (stiffness). An optimised result is given in the end.

\subsection{Results of laminate optimisation without further modification}
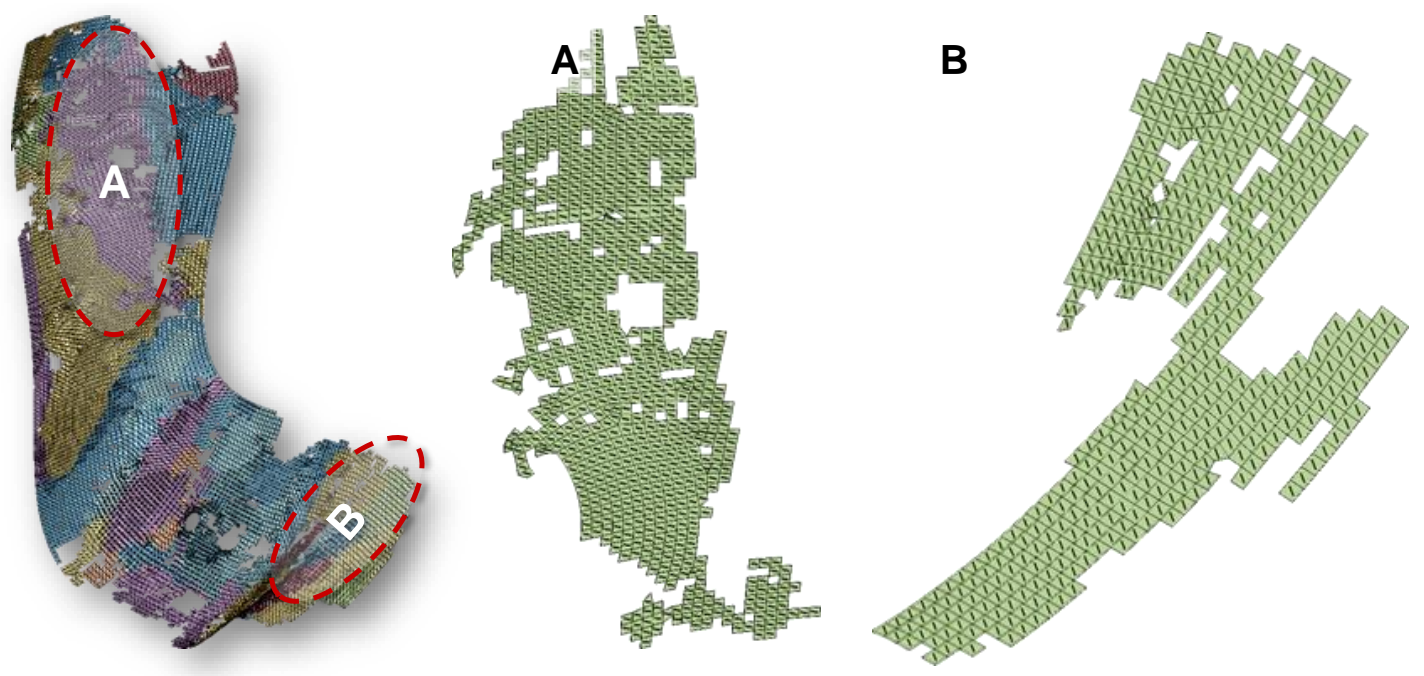

Figure 11. Laminate optimisation: theoretical results for glider seat without modification.

As discussed in the introduction, resulting ply geometries created by laminate optimisation approaches are frequently irregular in geometry and partly scattered (Figure 11) due to being optimised theoretically and underlying restrictions of the clustering algorithm. The clustering algorithm used here is bottom-up, i.e. similar directions from fibre orientation optimisation are grouped; for other laminate optimisation approaches it can be top-down, selecting from a range of possible fibre orientations. The latter seems to lead to less scattered ply geometry results (less holes), an example given in (Völkl et al., 2018a). However, outer contours remain heavily curved and thus unsuitable for ATL or AFP processes in later stages. Manual corrections are necessary in order to obtain manufacturable results. 


\subsection{Results with $75 \mathrm{~mm}$ unidirectional tape modification}
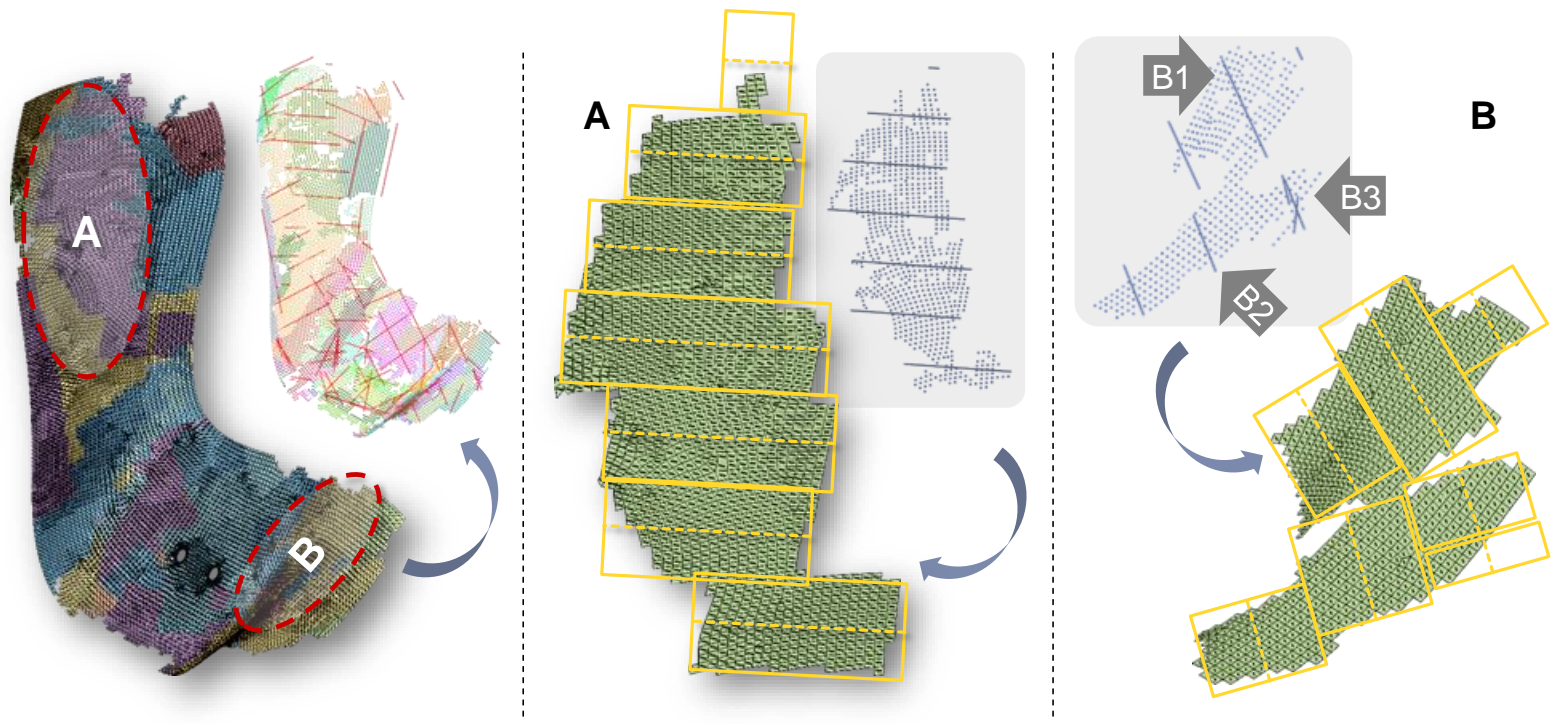

Figure 12. Modified optimisation results for the glider seat using $75 \mathrm{~mm}$ tape width

Applying the proposed algorithm with $75 \mathrm{~mm}$ tape width (normal vector tolerance 25 degrees), results become much more structured (Figure 12). The overall view of the tapes on the left side of Figure 12 provides an impression of necessary tape courses. Detail A shows a nearly-plane cluster, the same as in Figure 11 A, yet modified for ATL. The tape-laying algorithm produces seven parallel tape courses without interruption along the optimised fibre direction. In contrast, Detail B shows a ply geometry proposal being heavily bent in many directions and even exhibiting indents; thus sub-clustering using element normal vectors (subchapter 2.1) takes place, then the sub-clusters (one above $=\mathrm{B} 1$, one below=B2, some smaller ones=B3) individually get their tape courses (2.2), which after that are aligned to provide as-continuous-as-possible machining paths (2.3-2.4). Generally, there are still some challenges at part borders, e.g. at detail A on the top. The tape in this region exceeds the actual geometry region, which has to be corrected during further development. To keep the borders of the part intact, up to now the overhanging tape is simply assumed to be cut off. To conclude, Figure 13 provides an overview over all created ply geometries in an explosion view.

Without modification

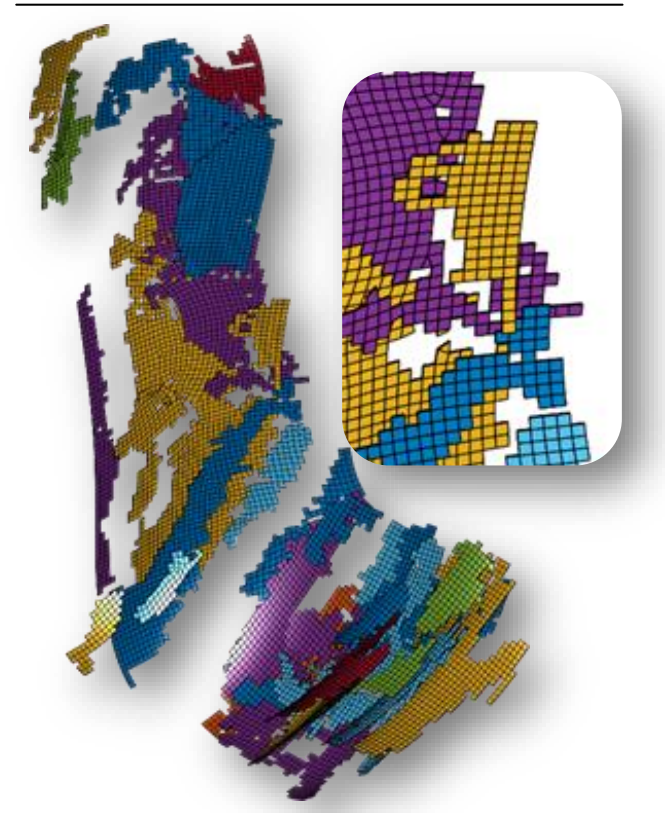

\section{Unidirectional tapes $(75 \mathrm{~mm})$}

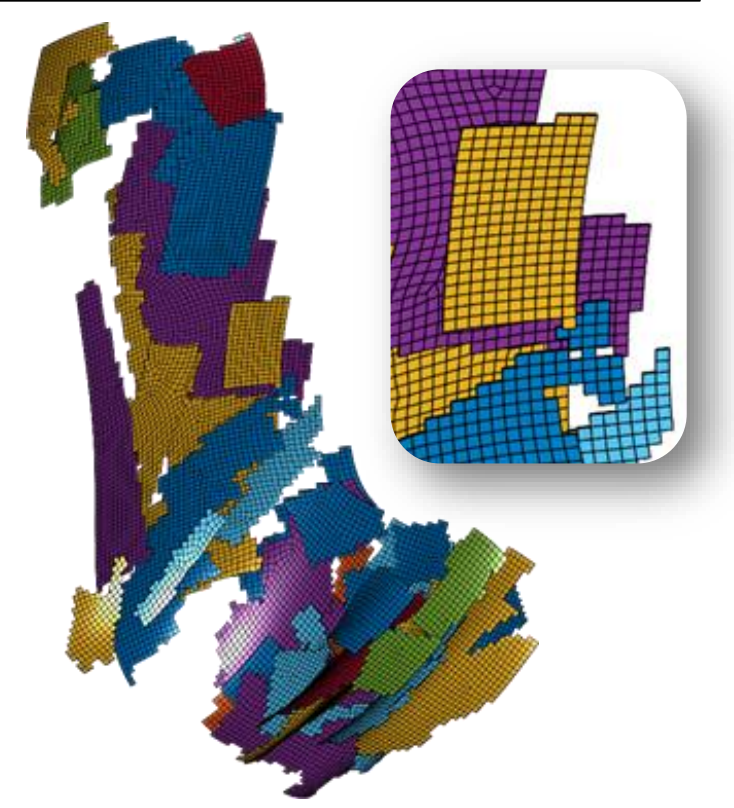

Figure 13. Overview of all modified ply geometries (left: before, right: after modification) 


\subsection{Resulting optimised model}

Meeting all constraints raised at the beginning of this chapter while minimising weight, the following overall result is generated by a MISQP optimisation algorithm, Figure 14. To obtain a more realistic Carbon Fibre Reinforced Plastics (CFRP) part, a (0/90)s stack-up forms a basic laminate; impact protection and shear stress absorption is realised by 45 degrees woven plies on top and bottom.
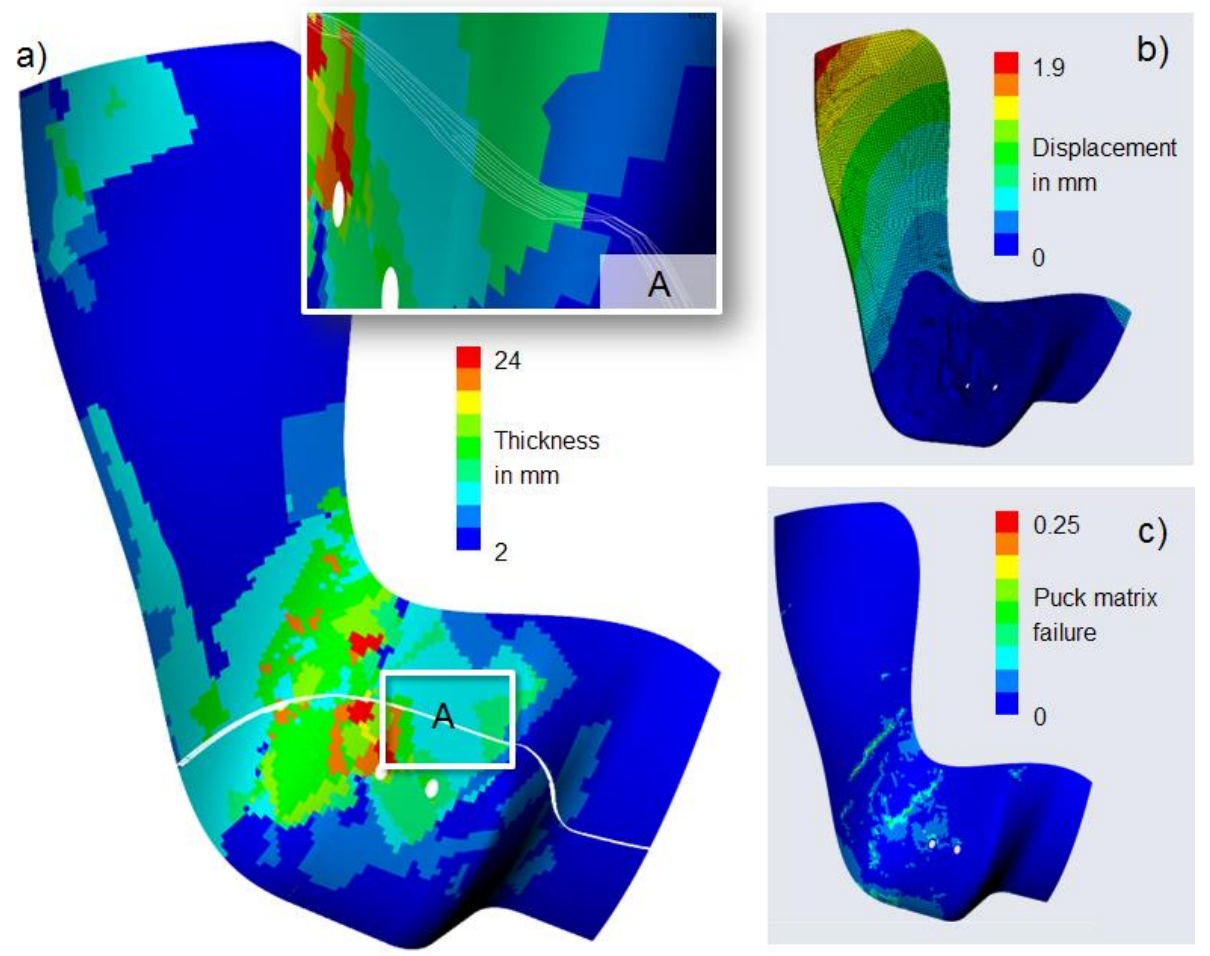

Figure 14. Resulting design meeting all constraints, a) Overall laminate thickness \& detail view, b) Puck matrix failure criterion, c) Overall displacement

The resulting design with $\sim 3 \mathrm{~kg}$ shows typical rectangular ply shapes (overall laminate thickness on the left, Figure 14a). The symmetric laminate (detail A) fulfils the displacement constraint "below 2 mm" with $1.9 \mathrm{~mm}$, which is also the critical constraint, Figure 14b). At this stiffness level, the failure criterion is below 0.25 (far below the 0.5 demanded, Figure 14c). Obviously, the stiffness criterion is stricter than the strength criterion, determining overall mass and laminate thickness of the part. All over the part, the matrix failure criterion was higher than fibre failure, therefore the evaluation only considers Puck matrix criterion. Locally, the wall thickness is very high, even for thickness capabilities (Lamontia et al., 2003) of ATL manufacturing. This of course can be improved, e.g. by using a sandwich structure.

\section{CONCLUSIONS AND AN OUTLOOK ON THE CHALLENGES OF FATIGUE IN LAMINATES}

The ATL/AFP modification method enhances laminate optimisation approaches and is a first step towards more manufacturability, even though operational questions remain open, such as the actual conversion to machine code and associated alterations. Anyway, it produces a proper and wellorganised laminate stackup with coherent, regularly shaped plies. However, enhancing laminate optimisation approaches does not necessarily need to be limited to manufacturing questions. In a more holistic view, addressing "Design-for-X"-relevant challenges becomes necessary: design for dynamic loads, e.g. crash, as CFRP parts may be used more and more in automotive design as soon as costs become increasingly reasonable; integration of functions, such as inserts or sensors; recycling topics and design for natural fibres; and fatigue.

Laminate optimisation results from FE simulations up to now stem from a nominal view of design: perfect, potentially pre-optimised geometry (Völkl et al., 2018b), perfect fibre orientation angles (from ideal draping), constant fibre volume fraction in all areas, constant prepreg thicknesses etc. This 
idealisation comprises a perfectly symmetric laminate as well, to avoid coupling and thus warping as good as possible. At the time of manufacturing, many of these assumptions prove wrong, especially when using manual techniques. Such uncertainties can be accounted for to some degree, e.g. by methods of robust design (Kellermeyer et al., 2017). Yet another challenge, however, which needs further scrutiny, is fatigue of FRP laminates. Over lifetime, the properties even of well-built laminates can change drastically due to fatigue, leading to undesired local material properties alterations.

Recent experimental investigations on fatigue properties of CFRP laminates scrutinise stiffness and strength degradation over a number of load cycles N. Figure 15 presents a qualitative stiffness degradation curve for a quasi-isotropic laminate, which occurs similarly in (He et al., 2016; Wu and Yao, 2010; Haefele and Herrera, 2015). It comprises three regions: as steep gradient of stiffness loss in the beginning (I), low and close-to-linear stiffness degradation (II) and a final, drastic stiffness decrease until fracture at the end (III) (Haefele and Herrera, 2015).

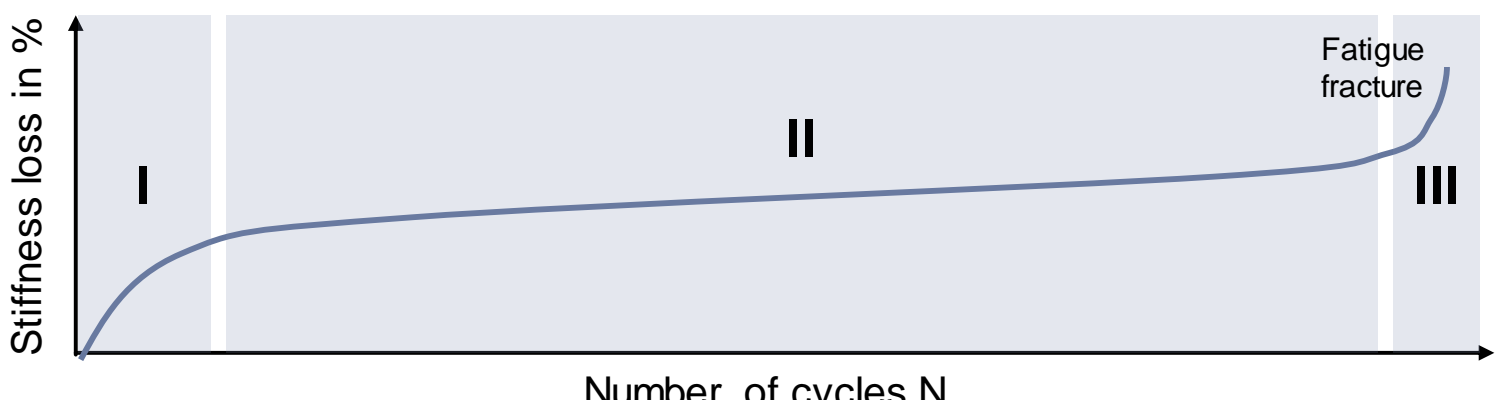

Figure 15. Qualitative stiffness degradation curve

In (Haefele and Herrera, 2015), the initial, rapid stiffness decrease (I) amounts to about $25 \%$ at a stress amplitude of $200 \mathrm{MPa}$ (stress ratio $\mathrm{R}=0.1$ ) and around 100.000 load cycles, which is considerable and will heavily influence the macroscopic part behaviour if a part is subjected to cyclic loading. Thinking of much more complicated laminate stack-ups as presented above, the case is even more difficult: locally within the laminate, stress intensity is most probably distributed inhomogeneously. In terms of strength, this might lead to some regions failing earlier than others due to fatigue. In less critical cases, thereof can simply result a relatively uncritical redistribution of loads; yet, in highly stressed laminates, even complete part failure.

Furthermore, regarding stiffness, with some layers failing while others remain, a previously symmetric laminate can become asymmetric, which can lead to warping due to coupling in the laminate (Schürmann, 2007) causing functional problems, like undesired contact between parts and instability. Calculation of remaining number of cycles until failure given a previous load spectrum is highly complicated. Thus, extending existing Computer Aided Engineering (CAE)-tools for laminate optimisation by this functionality is highly desirable for future applications involving cyclic loading, including many products made from FRPs, e.g. in automotive or aeroplane applications.

\section{REFERENCES}

Grimshaw, M.N., Grant, C.G. and Diaz, J. (2001), “Advance Technology Tape Laying for Affordable Manufacturing of Large Composite Structures”, in 46th International SAMPE Symposium, CRC Press, pp. 2484-2494.

Haefele, P. and Herrera, O. (2015), "Strength and stiffness degradation of carbon fiber reinforced plastic under cyclic loading under membran-, shear- and bending loading", Materials Science Forum, pp. 825-826, pp. 968-975. http://doi.org/10.4028/www.scientific.net/MSF.825-826.968.

He, M., Yang, T., Niu, X. and Du, Y. (2016), "Experimental investigation of the three-point bending fatigue properties of carbon fiber composite laminates", Advances in Material Science, Vol. 1 No. 1, p. 1. http://doi.org/10.18686/ams.v1i1.1.

Kellermeyer, M., Klein, D. and Wartzack, S. (2017), "Robust design of endless-fiber reinforced lightweight structures [Robuste Auslegung endlosfaserverstarkter Leichtbaustrukturen]”, Konstruktion, Vol. 69 No. 7-8, pp. 70-75.

Klein, D., Malezki, W. and Wartzack, S. (2015), "Introduction of a computational approach for the design of composite structures at the early embodiment design stage", Paper Presented at International Conference on Engineering Design (ICED15), 27.-30.07.2015, Milan, Italy. 
Lamontia, M., Funck, S., Gruber, M., Cope, R., Waibel, B. and Gopez, N. (2003), "Manufacturing flat and cylindrical laminates and built up structure using automated thermoplastic tape laying, fiber placement, and filament winding", SAMPE J (Soc Adv Mater Process Eng), Vol. 39 No. 2, pp. 30-38.

Lengsfeld, H., Wolff-Fabris, F., Krämer, J., Lacalle, J. and Altstädt, V. (2015), Faserverbundwerkstoffe: Prepregs und ihre Verarbeitung, Hanser, München.

Lukaszewicz, D.H.-J., Ward, C. and Potter, K.D. (2012), “The engineering aspects of automated prepreg layup. History, present and future", Composites Part B: Engineering, Vol. 43 No. 3, pp. 997-1009. http://doi.org/10.1016/j.compositesb.2011.12.003.

Reden, T.V., Schueppel, D. and Hohmann, A. (2018), "Development of production costs of CFRP parts", Paper Presented at ECCM18 - 18th European Conference on Composite Materials, 24.6.-28.6.2018, Athens, Greece (accessed 22 August 2018).

Schürmann, H. (2007), Konstruieren mit Faser-Kunststoff-Verbunden, 2nd ed., Springer, Heidelberg.

Völkl, H., Franz, M. and Wartzack, S. (2018a), “A case study on established and new approaches for optimized laminate design”, in ECCM18 - 18th European Conference on Composite Materials, pp. 1-8.

Völkl, H., Klein, D., Franz, M. and Wartzack, S. (2018b), “An efficient bionic topology optimization method for transversely isotropic materials”, Composite Structures, Vol. 204, pp. 359-367. http://doi.org/10.1016/j.compstruct.2018.07.079.

Wu, F. and Yao, W. (2010), “A fatigue damage model of composite materials", International Journal of Fatigue, Vol. 32 No. 1, pp. 134-138. http://doi.org/10.1016/j.ijfatigue.2009.02.027. 